\begin{tabular}{|c|l|}
\hline Title & Psychophysics of the probability weighting function \\
\hline Author(s) & Takahashi, Taiki \\
\hline Citation & $\begin{array}{l}\text { PhysicaA : Statistical Mechanics and its A pplications, 390(5), 902-905 } \\
\text { https://doi.org/10.1016/.physa.2010.10.004 }\end{array}$ \\
\hline Issue Date & 2011-03-01 \\
\hline Doc URL & http://hdl.handle.net/2115/45059 \\
\hline Type & article (author version) \\
\hline File Information & PhyA 390-5_902-905.pdf \\
\hline
\end{tabular}

Instructions for use 


\title{
Psychophysics of the probability weighting function.
}

\author{
Taiki Takahashi ${ }^{1}$
}

${ }^{1}$ Direct all correspondence to Taiki Takahashi (Email taikitakahashi@gmail.com)

Department of Behavioral Science, Faculty of Letters, Hokkaido University

N.10, W.7, Kita-ku, Sapporo, 060-0810, Japan

TEL +81-11-706-3057, FAX +81-11-706-3066.

Acknowledgements: The research reported in this paper was supported by a grant from the Grant- in-Aid for Scientific Research (Global COE) from the Ministry of Education, Culture, Sports, Science and Technology of Japan. 


\begin{abstract}
A probability weighting function w(p) for an objective probability $\mathrm{p}$ in decision under risk plays a pivotal role in Kahneman-Tversky's prospect theory. Although recent studies in econophysics and neuroeconomics widely utilized probability weighting functions, psychophysical foundations of the probability weighting functions have been unknown. Notably, a behavioral economist Prelec (1998) axiomatically derived the probability weighting function: $w(p)=\exp \left(-(-\ln p)^{\alpha}\right)(0<\alpha<1 ; w(0)=1, w(1 / e)=1 / e$, $\mathrm{w}(1)=1$ ), which has extensively been studied in behavioral neuroeconomics. The present study utilizes psychophysical theory to derive Prelec's probability weighting function from psychophysical laws of perceived waiting time in probabilistic choices. Also, the relations between the parameters in the probability weighting function and the probability discounting function in behavioral psychology were derived. Future directions in the application of the psychophysical theory of probability weighting function in econophysics and neuroeconomics are discussed.
\end{abstract}

Keywords: Neuroeconomics; Econophysics; Uncertainty; Prospect theory; Decision-making; Tsallis' statistics 


\section{Introduction:}

Humans and non-human animals devalue probabilistic rewards as the receipt becomes more uncertain. The preference for a certain reward over an uncertain reward of an equal expected value is referred to as risk aversion in decision-making under risk/uncertainty. In von Neumann-Morgenstern's expected utility theory widely adopted in game theory, risk aversion is represented by a concavity of the utility function (von Neumann and Morgenstern, 1947). The expected utility theory is a normative theory of rational decision-making under risk which satisfies a set of rationalizable axioms such as the independence axiom (von Neumann and Morgenstern, 1947). However, later empirical and experimental studies revealed that human subjects often violate the rationality in decision under risk (e.g., Allais paradox, see Allais, 1953). In their seminal paper on prospect theory, Kahneman and Tversky (1979) explained the observed violations of rationality in decision under risk by introducing a nonlinear transformation of objective probabilities into “decision weights" $\mathrm{p} \rightarrow \mathrm{w}(\mathrm{p})$ for $\mathrm{p}, \mathrm{w}(\mathrm{p}) \in[0,1]$, where $\mathrm{p}$ is the probability of winning a reward and $w(p)$ is the probability weighting function determining the decision weight for the probability and satisfying $w(0)=0$ and $w(1)=1$. Furthermore, a behavioral economist Prelec derived, from preference axioms, the probability weighting function:

$$
\mathrm{w}(p)=\exp \left(-(-\ln p)^{\alpha}\right)
$$

where $\alpha \in[0,1]$ is a free parameter determining the nonlinear distortion of the subjective probability in decision under risk (Prelec, 1998). We can see that Prelec's probability weighting function satisfies $w(0)=1, w(1 / e)=1 / e$ and $w(1)=1$ (i.e., 1/e is a fixed point). Note that the subjective value of the uncertain reward $V(x, p)$ is

$$
\mathrm{V}(\mathrm{x}, \mathrm{p})=\mathrm{v}(\mathrm{x}) \mathrm{w}(\mathrm{p}),
$$

where $\mathrm{v}(\mathrm{x})$ is the value of the reward $\mathrm{x}$, and $\mathrm{p}$ is the probability of winning the reward. In expected utility theory, $w(p)=p$ (i.e., linear probability weighting). In analyzing risk aversion in economic transactions, a simple q-probability weighting function developed in deformed algebra inspired by Tsallis' thermodynamics $\left(w(p)=p^{q}\right)$ has been introduced in econophysics (Anteneodo et al., 2002). In recent neuroeconomic studies, neural correlates of Prelec's probability weighting function has extensively been studied. Paulus and Frank (2006) reported that $\alpha$ in equation 1 is correlated with the neural activity in the anterior cingulate in the human brain, and Hsu et al. (2009) at Camerer's 
group revealed that neural representation of probabilities is nonlinear, by utilizing Prelec's probability weighting function (equation 1). Therefore, it is of importance to examine psychophysical foundations of the probability weighting functions for a better understanding of neuropsychological processing underlying decision under risk, and future applications of the probability weighting function in econophysics.

I derive, in this paper, the widely-employed Prelec's probability weighting function as a special case, from psychophysical laws of perceiving waiting time in probabilistic choices in decision under risk. The psychophysical theory is relevant to the q-exponential probability discounting model based on deformed algebra inspired by Tsallis' statistics (Takahashi, 2007). Finally, it is demonstrated that the parameters in the probability weighting function and the hyperbolic probability discounting model in behavioral psychology (Rachlin et al., 1991) are mathematically related.

This paper is organized in the following manner. In Section 2, I briefly introduce psychophysics of the q-exponential probability discount model based on Tsallis' statistics. This model is based on psychophysical equivalence of delay in intertemporal choice and waiting time in probabilistic choice. In Section 3, I derive the probability weighting function based on psychophysical laws of perceived waiting time in probabilistic choices with probability weighting or physical probability. I also demonstrate mathematical relations between parameters in Prelec's probability weighting function and a general probability discounting function. In Section 4, some conclusions from this study and future study directions by utilizing the psychophysical theory of the probability weighting function related to the q-exponential probability discounting, in neuroeconomics and econophysics are discussed.

\section{Psychophysics of q-exponential probability discounting}

\subsection{A mathematical equivalence of delay and uncertainty in decision making}

Rachlin and colleagues proposed that delay until receipt of gains in intertemporal choice and uncertainty of winning of gains in probabilistic choice may be equivalent (Rachlin et al., 1991). In this theory, a decrease in a probability of winning an uncertain reward corresponds to an increase in a delay until winning the reward. Specifically, an average waiting time until winning an uncertain reward is proportional to $(1 / p)-1$ ("odds against" $=\mathrm{O}_{\mathrm{p}}$ ), where $p$ is a probability of winning the uncertain reward. Therefore, decision-making models in intertemporal choice can straightforwardly be extended into probabilistic choice, after replacing a parameter of delay in intertemporal choice models with the odds against parameter.

Recently, the following time-discount function (q-exponential discount 
function) for intertemporal choice has been proposed by Cajueiro (2006) and empirically examined in our previous study (Takahashi et al., 2007):

$$
V(D)=V(0) / \exp _{q}(k D)=V(0) /[1+(1-q) k D]^{1 /(1-q)}
$$

where $\mathrm{V}(\mathrm{D})$ is a discounted value of the delayed reward obtained at delay D. $\exp _{q}()$ is the q-exponential function in the deformed algebra inspired by Tsallis' non-extensive thermodynamics, $q<1$ is a consistency parameter, and $k$ is an impulsivity parameter; i.e., larger $\mathrm{k}$ values indicate stronger preference for smaller sooner reward over larger later reward. Larger q $(<1)$ values correspond to more consistent intertemporal choice; namely, $q \rightarrow 1$ corresponds to exponential discounting (complete consistency), while $q=0$, hyperbolic discounting (complete inconsistency) (Cajueiro, 2006; Takahashi et al., 2007). Takahashi (2007) introduced the q-exponential probability discounting model by putting the odds against $\mathrm{O}_{\mathrm{p}}=(1 / \mathrm{p})-1$ (which is proportional to waiting time in probabilistic choices) instead of delay $\mathrm{D}$ in equation 3 :

$$
\begin{aligned}
V(p) & =V(0) / \exp _{q}\left(k_{p}(1-p) / p\right) \\
& =V(0) /\left[1+k_{p}(1-q)(1-p) / p\right]^{1 /(1-q)}
\end{aligned}
$$

where $p$ is the probability of winning an uncertain reward and $k_{p}$ is a parameter of delay aversion in repeated probabilistic choices; i.e., larger $k_{p}$ values indicate stronger risk (i.e., delay until winning) aversion (other parameters have the same definitions in Equation 3).

\subsection{Psychophysics of waiting time in probabilistic choices}

Takahashi (2005) proposed that psychological time $\tau$ in intertemporal choice is logarithmic in physical time D (i.e., Weber-Fechner law),

$\tau(\mathrm{D})=\mathrm{a}_{\mathrm{t}} \ln \left(1+\mathrm{b}_{\mathrm{t}} \mathrm{D}\right)$

where $a_{t}$ and $b_{t}$ are free positive parameters. The logarithmic time may be utilized by an agent in intertemporal choice to discount delayed rewards exponentially with psychological time $\tau$, resulting in non-exponential discounting in physical time (Takahashi, 2005). This proposal has later been confirmed experimentally by a behavioral economic study which measured psychological time directly (Zauberman et al., 2009). Let us suppose, as a natural assumption, that "subjective waiting time in probabilistic choices” $\left(:=\tau_{\mathrm{p}}\right)$ is also logarithmic in "physical waiting time” $\left(:=\mathrm{D}_{\mathrm{p}}\right)$ which 
is proportional to “odds against in terms of physical probability” $\left(=\mathrm{O}_{\mathrm{p}}\right)$ :

$\tau_{p}\left(O_{p}\right)=a_{p} \ln \left(1+b_{p} t_{p} O_{p}\right)=a_{p} \ln \left[1+b_{p} t_{p}(1 / p-1)\right]$

where $a_{p}$ and $b_{p}$ are positive free parameters, and $t_{p}$ is the average inter-trial time of probabilistic choices (i.e., delay $\mathrm{D}_{\mathrm{p}}=\mathrm{t}_{\mathrm{p}} \mathrm{O}_{\mathrm{p}}$, referred to as "physical weighting time"). Notably, exponential discounting with the logarithmic waiting-time perception is mathematically equivalent to the q-exponential probability discounting (equation 4) based on Tsallis' statistics.

Let us further suppose that "subjective waiting time in terms of subjective probability (i.e., the probability weighting function)" $\left(:=\tau_{\mathrm{w}}\right)$ is also logarithmic in "odds against in terms of the probability weighting function” $\left(:=\mathrm{O}_{\mathrm{w}}\right)$ :

$\tau_{\mathrm{w}}\left(\mathrm{O}_{\mathrm{w}}\right)=\mathrm{a}_{\mathrm{w}} \ln \left(1+\mathrm{b}_{\mathrm{w}} \mathrm{t}_{\mathrm{w}} \mathrm{O}_{\mathrm{w}}\right)=\mathrm{a}_{\mathrm{w}} \ln \left[1+\mathrm{b}_{\mathrm{w}} \mathrm{t}_{\mathrm{w}}(1 / \mathrm{w}(\mathrm{p})-1)\right]$

where $a_{w}$ and $b_{w}$ are positive free parameters, and $t_{w}$ is the average inter-trial time of probabilistic choices in terms of probability weighting w(p) (i.e., delay in terms of w(p) is $\mathrm{D}_{\mathrm{w}}=\mathrm{t}_{\mathrm{w}} \mathrm{O}_{\mathrm{w}}$, referred to as "probability-weighted waiting time").

\section{Psychophysical theory of the probability weighting function}

\subsection{Psychophysical laws on perception of physical and probability-weighted waiting time}

Let us now assume that a relation between perceived "physical waiting time" and perceived "probability-weighted waiting time” follows Stevens' power law (introduced by Takahashi (2006) for "subadditivity” in temporal discounting):

$$
\tau_{\mathrm{w}}\left(\mathrm{O}_{\mathrm{w}}\right)=\tau_{\mathrm{p}}\left(\mathrm{O}_{\mathrm{p}}\right)^{\mathrm{s}}(\mathrm{s} \in[0,1])
$$

Equivalently,

$a_{w} \ln \left[1+b_{w} t_{w}(1 / w(p)-1)\right]=\left[a_{p} \ln \left[1+b_{p} t_{p}(1 / p-1)\right]\right]^{s}$

It is to be noted that equation 5 denotes the relation between physical and psychological time; while equation 8 denotes the relation between two distinct types of psychological times. Therefore, we assume Stevens' power law in equation 8 , rather than a logarithmic relation. 
By solving equation 9 in terms of w(p), we obtain

$\mathrm{w}(\mathrm{p})=\frac{b_{w} t_{w}}{\exp \left[a_{w}{ }^{-1} a_{p}^{s}\left\{\ln \left[1+b_{p} t_{p}\left(p^{-1}-1\right)\right]\right\}^{s}\right]+b_{w} t_{w}-1}$.

We can see that $\mathrm{w}(0)=0$ and $\mathrm{w}(1)=1$, indicating that $\mathrm{w}(\mathrm{p})$ in equation 10 is the generalized probability weighting function for prospect theory. If we now assume that $a_{w}^{-1} a_{p}^{s}=b_{p} t_{p}=b_{w} t_{w}=1$, we obtain

$\mathrm{w}(p)=\exp \left(-(-\ln p)^{\mathrm{s}}\right)$

which is equivalent to equation 1 (Prelec's probability weighting function). Therefore, the nonlinearity parameter in Prelec's probability weighting function is an exponent of Stevens' power law of phychophysics of waiting time (equation 8). Finally, when $b_{w} t_{w}=a_{w}{ }^{-1} a_{p}^{s}=\mathrm{s}=1$ are assumed in equation 10 , we obtain,

$$
\mathrm{w}(\mathrm{p})=\frac{1}{\left[1+b_{p} t_{p}(1 / p-1)\right]},
$$

which is the hyperbolic probability discounting function (Rachlin et al., 1991). Taken together, it can be said that Prelec's one-parameter probability weighting function is the simplest special case of the general probability weighting function (equation 10), which is derived from psychophysical theory relevant to deformed algebra inspired by Tsallis' thermodynamics.

\section{Conclusions and implications for neuroeconomics and econophysics}

This study is the first to demonstrate that Prelec's probability weighting function is a simple reflection of psychophysics of waiting-time perception in probabilistic choices. A recent mathematical psychological study discovered that the Prelec's probability weighting function can also be derived from the power-invariance principle:

$w\left(p^{q}\right)=(w(p))^{f(q)}$ 
where $\mathrm{q}$ is a free parameter and $\mathrm{f}(\mathrm{q})$ is independent of $\mathrm{p}$ (Al-Nowaihi and Dhami, 2006). This invariance principle may also be relevant to q-probability in theory of economic transactions (Anteneodo et al., 2002). Also, because parameter in Prelec's probability weighting function is associated with neural activities in the anterior cingulate cortex (Paulus et al., 2006) and the striatum (Hsu et a., 2009), what types of psychophysical computation is performed in these neural networks should be examined in future studies for a better understanding of the role of time-perception in decision under risk (probabilistic choices). Furthermore, future statistical physical studies should examine how the power-invariance principle and psychophysical laws are related, by utilizing more general frameworks such as renormalization theory. 


\section{References}

Allais, M., (1953), Le comportement de l'homme rationnel devant le risque: critique des postulats et axiomes de l'école Américaine, Econometrica 21, 503-546.

A Al-Nowaihi, S Dhami (2006) A simple derivation of Prelec's probability weighting function Journal of Mathematical Psychology, Volume 50, Issue 6, Pages 521-524

Anteneodo C, Tsallis C, Martinez AS. Risk aversion in economic transactions. 2002 Europhysics Lett. 59 (5): 635-641.

Cajueiro D.O. A note on the relevance of the q-exponential function in the context of intertemporal choices. Physica A 364 (2006) 385-388.

Hsu M, Krajbich I, Zhao C, Camerer CF. (2009) Neural response to reward anticipation under risk is nonlinear in probabilities. $\boldsymbol{J}$ Neurosci. 2009 Feb 18;29(7):2231-2237.

Von Neumann, J., and O. Morgenstern (1947): Theory of Games and Economic Behavior, 2nd ed. Princeton: Princeton University Press.

Paulus MP, Frank LR. (2006) Anterior cingulate activity modulates nonlinear decision weight function of uncertain prospects. Neuroimage. 1;30(2):668-677.

Prelec D (1998) The probability weighting function. Econometrica, Vol. 66, No. 3, pp. 497-527

Rachlin H, Raineri A, Cross D. (1991) Subjective probability and delay. J Exp Anal Behav. 55(2):233-244.

Takahashi T. (2005) Loss of self-control in intertemporal choice may be attributable to logarithmic time-perception. Med Hypotheses. 65(4):691-693.

Takahashi T (2006), “Time-Estimation Error Following Weber-Fechner Law May Explain Subadditive Discounting,” Medical Hypotheses, 67 (6), 1372-1374. 
Takahashi (2007) A probabilistic choice model based on Tsallis' statistics. Physica A: 386, 335-338.

Takahashi T, Ono H, Radford MHB (2007) Empirical estimation of consistency parameter in intertemporal choice based on Tsallis' statistics Physica A 381: 338-342

von Neumann, J., \& Morgenstern, O. (1947). Theory of games and economic behavior, 2nd ed. Princeton, NJ: Princeton University Press.

Gal Zauberman, B. Kyu Kim, Selin Malkoc, James R. Bettman (2009), Discounting Time and Time Discounting: Subjective Time Perception and Intertemporal Preferences, Journal of Marketing Research, 46(4). 543-556. 